\title{
ADIABATIC APPROXIMATION IN A RESONANCE CAPTURE PROBLEM
}

\author{
L.A. KALYAKIN
}

\begin{abstract}
By means of the averaging method, we analyze two model problems on capture into resonance that leads us to the adiabatic approximation in the leading term in the asymptotics. The main aim is an approximate (by using a small parameter) description of the domain of capture into resonance. This domain is in the phase plane and it is formed by the initial points for the resonance solutions with an unboundedly increasing energy. The capture domain depends on an additional parameter involved in the equation. We show that the adiabatic approximation fails as the capture domain becomes narrow. In this case we have to modify substantially the averaging method. As a result, a system of nonlinear differential equations arises for the leading term in the asymptotics and this system is not always integrable.
\end{abstract}

Keywords: nonlinear oscillations, small parameter, asymptotics, capture into a resonance, adiabatic approximation.

Mathematics Subject Classification: 34D10, 34D20, 37J25, 34E13

\section{INTRODUCTION}

The opinion on an exceptional efficiency of mathematical methods in scientific studies is current and makes an impression of an all-powerfull mathematics. A wide use of computers encourages the spreading of this myth. Meanwhile, in applied studies, the limited possibility of mathematical approach often arises. The arising problems are usually explained by disadvantages in the employed models, either by excessive complicacy or by excessive simplicity. The efficiency of the mathematics in applications is mostly based on the art of simplifying complicated models to a reasonable level. In the most clear way this is reflected in problems posed in the form of differential equations. original complicated equations are replaced by equations more simple and admissible for an analytic and numerical analysis. An accurate mathematical formalization of simplification is made by involving the notion of asymptotic approximation [1], 2]. Such approach is not always simple. The difficulties one has to overcome are related with non-uniformness of the asymptotics [3]. Sometimes, the provided asymptotic formulae are not applicable in one or another domain. For instance, in the trivial example $\exp \left(-\frac{x}{\varepsilon}\right)$ the asymptotic approximation $\exp \left(-\frac{x}{\varepsilon}\right) \approx 0$ as $\varepsilon \rightarrow 0$ is apriori not applicable in the sector $\frac{x}{\varepsilon} \leqslant 1$. The non-uniformities in asymptotic expansion arise for the functions of several variables and usually indicate the presence of essentially singular points.

While studying the solutions to differential equations, the expansions in a small parameter are widely used. Since apart of the parameter, the solution depends on at least on independent variable, in such problems one often faces with the problem of non-uniform expansions.

L.A. Kalyakin, Adiabatic approximation in a resonance capture problem.

(c) KALYAKin L.A. 2017.

The research is supported by the grant of Russian Science Foundation (project no. 17-11-01004).

Submitted April 2, 201\%. 
Various methods based on the idea of the many scales were developed for constructing uniform asymptotic expansions [3]-[5]. At that, the most part of the researchers prefer to deal with expansions in one scalar parameter. The presence of more independent parameters leads one to additional problems with the uniformity, which are seldom discussed. However, this direction of the studying is interesting for applications and contains new mathematical problems, see, for instance, [6].

In this problem we analyze the problem on capture into resonance under the presence of two parameters in the original equations. The expansion in one of the parameters create an illusion that the other parameter is inessential. The invalidation of such illusion is shown by two examples.

1.1. Formulation of the problem. While analyzing resonance effects in dynamical systems, one of the targets is the domain of the capture into the resonance, which is the initial points for the resonance trajectories. For nonlinear systems this set, as a rule, does not coincide with the entire phase space. A character example is provided by the system of two equations

$$
\frac{d \rho}{d t}=\alpha \sin \varphi, \quad \frac{d \varphi}{d t}=\lambda-\rho, \quad t>0
$$

In asymptotic constructions giving rise to these equation, the functions $\rho(t)$ and $\varphi(t)$ are interpreted as a corrector for the energy and the phase shift of fast oscillations. The resonance solutions are identified with an unboundedly increasing function $\rho(t)$ and a bounded phase $\varphi(t)$. Such solutions can exist for several parameters, say, as $\lambda=\mu t, \mu, \alpha=$ const $>0$ [7]. In a more general case $\lambda=\lambda_{0}+\mu t$, the constant $\lambda_{0}$ is excluded by a shift in the variable $\rho$. The differences between resonance and non-resonance solutions are shown in Figure 1 .
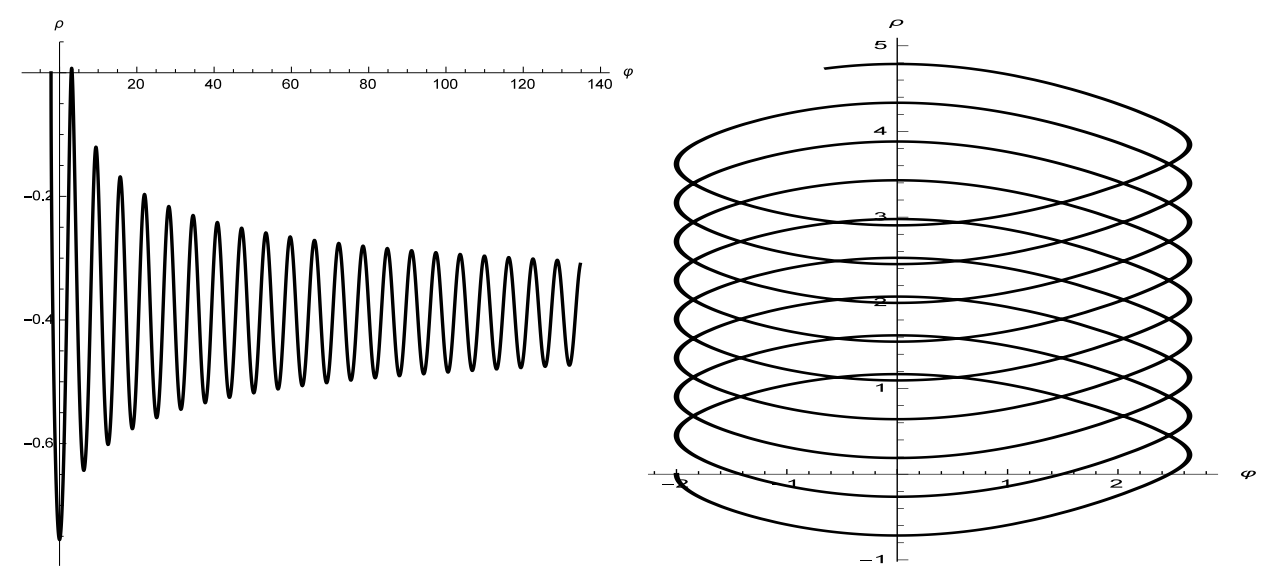

FiguRE 1. Numerical solution of equation (1). The parameters are $\mu=0.02$, $\alpha=0.2$. The left trajectory is of the non-resonance solution with the initial point $(-2.2,0)$. The right trajectory is of the resonance solution with the initial point $(-2,0)$

The capture domain for the case $\lambda=\mu t$ is given in the phase plane with the Cartesian coordinates $(\varphi, \rho)$, see Figure 2. This set is described by inequalities (6), see Section 2, In view of the relations in (6), we see that as $\mu \rightarrow 0$, the capture domain is extended to the maximal possible one bounded by the separatrices of the mathematical pendulum. And as $\alpha \rightarrow 0$, this domain becomes smaller and disappears at all as $|\alpha|<|\mu|$. Such result is possible thanks to the integrability of the system as $\lambda=\mu t$. 


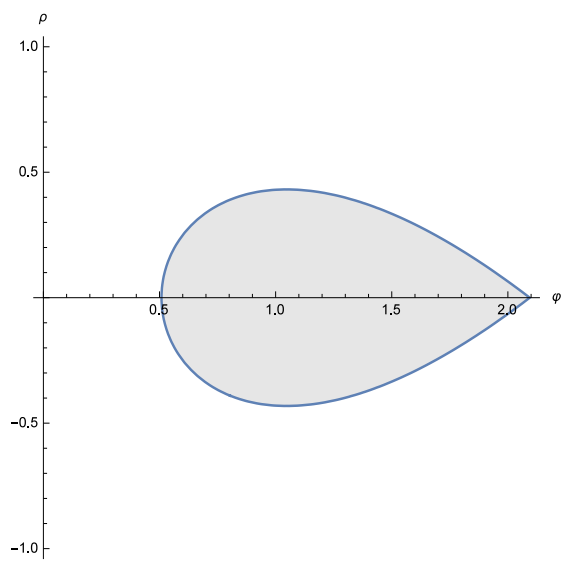

Figure 2. The domain of the capture into resonance as $\lambda=\mu t, \mu=\frac{\sqrt{3}}{2}, \alpha=1$

However, such explicit description of the capture domain is not always possible. A less trivial example is given by the system of main resonance equations:

$$
\frac{d r}{d t}=\alpha \cos \psi, \quad r\left[\frac{d \psi}{d t}+r^{2}-\lambda\right]=-\alpha \sin \psi, \quad t>0
$$

Here the variables $r, \psi$ are interpreted as the amplitude and the angle in polar coordinates in the plane. In the case $\lambda=\lambda_{0}+\mu t, \alpha, \mu, \lambda_{0}=$ const $>0$, the existence of resonance solutions can be proved by studying the asymptotics of the solutions at infinity. For the amplitude unboundedly growing in the leading term, we obtain $r(t)=\sqrt{\mu t}+\mathcal{O}(1), t \rightarrow \infty$. There exists a two-parametric family of such solutions [8]. However, we fail to identify the corresponding set of the initial data [9]. The situation becomes also worse because of the presence of a twoparametric solution of non-resonance solutions with a bounded amplitude. The impossibility of analytic description of the capture domain is explained sometimes by the non-integrability of the system (2), more precisely, by the absence of the connection formulae known, for instance, the Painlevé equations.

We note that in the above examples the growth of the amplitude is due to the non-autonomy of the equations. As $\lambda=$ const, each of the systems has a first integral and all solutions are bounded in the component $\rho(t)$ or $r(t)$. The integrability of the "frozen" problem can be employed for studying the capture domain in the particular case of a weak non-autonomy when $\lambda=\lambda_{0}+\mu t$ and the factor $\mu$ is small. The corresponding approaches are based on constructing the asymptotics in the small parameter $\mu \rightarrow 0$ and are usually related with the notion "adiabatic approximation" [5]. However, in the asymptotic constructions in a small parameter, there always rises the problem on uniformity in other parameters involved in the equation. In the considered systems the problems arise as $|\alpha| \ll 1$. For instance, in the first example the condition necessary for the existence of the capture domain imposes the restriction $|\mu / \alpha| \leqslant 1$, which means that $\alpha$ can not be too small.

The above examples looks rather simple. In these examples, the parameter $\alpha$ can be excluded by a scale transform reducing the examples to the case $\alpha=1$. It is clear that a new combination arises instead of $\mu$. For instance, in the first example there arises the quotient $\mu / \alpha$. In this case the adiabatic approximation makes sense only as $\mu / \alpha \rightarrow 0$.

In more complicated examples, it is impossible to get rid of additional parameters in such simple way. As an example, the following system of two differential equations serve:

$$
\gamma \frac{d \gamma}{d t}=\partial_{\psi} Z(\gamma, \psi), \quad \gamma \frac{d \psi}{d t}=-\partial_{\gamma} Z(\gamma, \psi), \quad t>0,
$$


defined by the function

$$
Z(\gamma, \psi) \equiv \frac{1}{2}(\gamma-\lambda)^{2}+\alpha \sqrt{\gamma^{2}+2 b \gamma-d} \sin \psi
$$

Here $b, d>0$ are fixed constants. The positive zero of the radicand in $\gamma_{0}=\sqrt{b^{2}+d}-b$ determines the boundary of the domain $D=\left\{\gamma>\gamma_{0}, \psi \in \mathbb{R}^{1}\right\}$, in which the problem is considered. The equations of form (3), (4) rise while describing the motion of a charged particle in the field of a plane electromagnetic wave running in the direction of a homogeneous magnetic field [10, Sect. 17], see also[11.

If $\lambda=$ const, system (3) is integrable and on each solution the component $\gamma(t)$ is bounded. In the non-autonomous case with $\lambda=\lambda_{0}+\mu t$ system (3) is not integrable. In the case of a weak non-autonomy with $|\mu| \ll 1$, there arise solutions with an unboundedly growing component $\gamma(t)[12$. However, now $\alpha$ is not excluded by scale transformation and it remains unclear what problem rise in the adiabatic approximation because of this additional parameter.

One should bear in mind that in the known works devoted to the analysis of system (3) the adiabatic approximation was not used at all. For instance, the construction proposed in [13, [14] does not correspond to the adiabatic approximation. At first glance, the ignoring of the adiabatic approximation for a weakly non-autonomous system looks strange and moreover, this leads to more complicated asymptotic constructions. Nevertheless, such ignoring was also in similar situations [9], 15.

In the present work we find out the relations for the parameters of the original system, under which the adiabatic approximation turns out to be ineligible for describing the domain of the capture into the resonance. The ineligibility is found for a small coefficient $\alpha$ and it is reflected in a narrow capture domain, which collapses into a line as $\alpha \rightarrow 0$. This phenomenon has no relation with the dynamical bifurcation saddle-center, which is often discussed in relation with the problem on capture into the resonance, see, for instance, [15]-[17]. The principal difference is that the collapsing of the capture domain corresponds to the bifurcation to a slipping line consisting of the equilibrium points.

\section{EXAMPLE OF A SHIFTED PENDULUM}

In order to clarify the role of the parameter $\alpha$ in the adiabatic approximation, it is useful to analyse a problem with a known explicit solution. As such problem, we consider the simplest nonlinear weakly non-autonomous problem (1). In this system, the small parameter $0<\mu \ll 1$ is involved in the coefficient $\lambda=\mu t$. The differential equations are completed by the initial conditions

We need the following.

$$
\left.\rho\right|_{t=0}=\rho_{0},\left.\quad \varphi\right|_{t=0}=\varphi_{0}
$$

1. Find conditions, under which there exist exact solutions with an unboundedly growing component $\rho(t)$, that is, to determine the exact domain of the capture into resonance.

2. Obtain an approximate description of the capture domain on the base of adiabatic approximation with the small parameter $\mu$.

3. Find the restrictions for the parameter $\alpha$, under which the adiabatic approximation become ineligible for describing the capture domain.

2.1. Exact solution. In the case $\lambda=\mu t$ system (1) with reduced to the equation of the mathematical pendulum with a constant torsional moment (shifted pendulum equation)

$$
\frac{d^{2} \varphi}{d t^{2}}=\mu-\alpha \sin \varphi
$$


well-known in the problems on the capture into resonance [5], [7]. The equation is integrable since there exists a first integral

$$
\frac{\dot{\varphi}^{2}}{2}-\mu \varphi-\alpha \cos \varphi=\text { const. }
$$

The information on possible solutions can be found in the phase portrait in the Cartesian coordinates $(\varphi, \dot{\varphi})$.

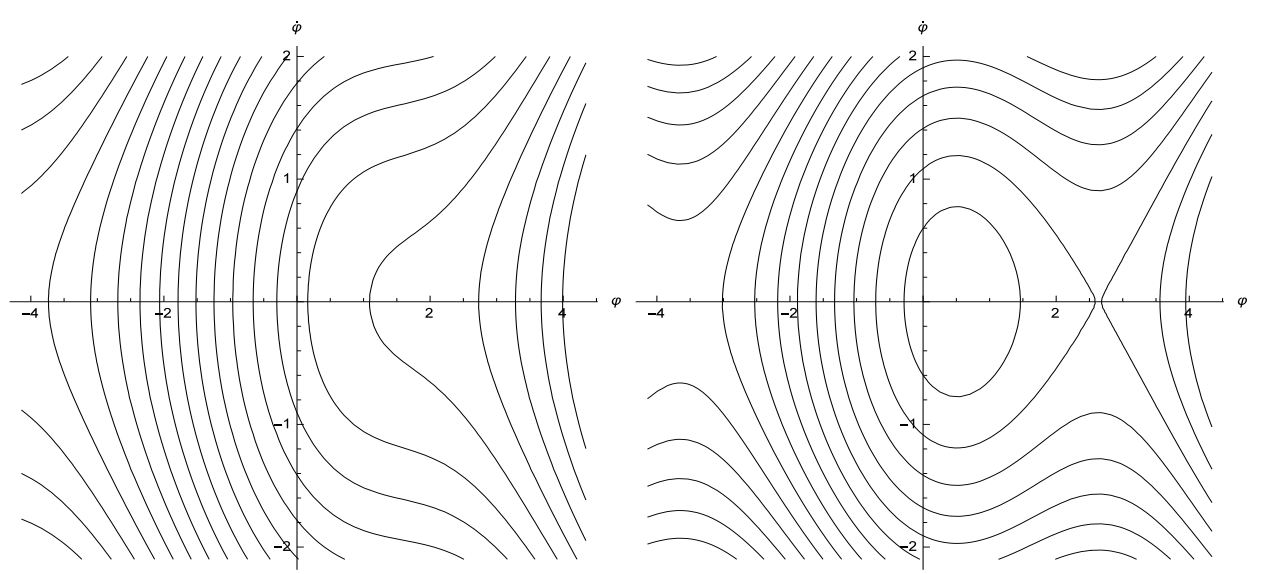

Figure 3. Phase portrait of the equation of the mathematical pendulum with a torsional moment. In the left: $|\mu / \alpha|>1$, in the right: $|\mu / \alpha|<1$

If $|\mu / \alpha|>1$, equation (5) has no stationary points and all trajectories go to infinity, see Figure 3. Such solutions with an unboundedly increasing component $\psi(t)=\mu t^{2}\left[\frac{1}{2}+\mathcal{O}\left(t^{-1}\right)\right]$, $t \rightarrow \infty$, correspond to the slipping. The second component $\rho(t)$ is bounded for such solutions.

If $|\mu / \alpha|<1$, equation (5) has stationary points of saddle and center type. The separatrix loop envelopes the center and forms the boundary of the domain, inside which the phase trajectories are closed and are associated with periodic solutions with a bounded phase $\psi(t)$, see the right part of Figure 3. Apart this, there are many solutions with slipping (an unbounded growth) of the phase.

These results obtained for equation (5) allow one to solve completely the issue on resonance solutions in system (1). Such solutions exist only under the condition $|\mu / \alpha| \leqslant 1$. Their initial values $\left(\varphi_{0}, \rho_{0}\right)$ are either inside the separatrix loop or lie on the separatrices coming to the saddle. The component $\varphi(t)$ of such solutions is bounded, while according to the second equation in (1), $\rho(t)$ grows:

$$
\rho(t)=\lambda(t)-\dot{\varphi}(t)=\mu t+\mathcal{O}(1), \quad t \rightarrow \infty .
$$

The analytic description of the original capture domain (the set of initial data $\left.\left(\varphi_{0}, \rho_{0}\right)\right)$ is given by the formulae:

$$
-\alpha \cos \varphi_{c}-\mu \varphi_{c} \leqslant \frac{1}{2} \rho_{0}^{2}-\alpha \cos \varphi_{0}-\mu \varphi_{0} \leqslant-\alpha \cos \varphi_{s}-\mu \varphi_{s} .
$$

Here $\varphi_{c}, \varphi_{s}$ are the coordinates of the stationary points of center and saddle type determined by the equation $\sin \varphi=\mu / \alpha$ with the conditions $0<\varphi_{c}<\varphi_{s}<\pi$. Because of $2 \pi$-periodicity in $\varphi$, we consider one loop in the phase plane.

As $\mu \rightarrow 0$, the limiting position of the capture domain is a cell bounded by the separatrices of the pendulum:

$$
-\alpha \leqslant \frac{1}{2} \rho_{0}^{2}-\alpha \cos \varphi_{0} \leqslant \alpha
$$


This domain can be employed as the approximation of the exact capture domain for small $\mu \ll 1$. However, such approximation is not uniform in $\alpha$. For small $\alpha \approx \mu \ll 1$, cell (7) can not be employed for approximate description of the capture domain because of large errors. For instance, as $\alpha<\mu$, there are no resonance solutions at all, while cell (7) has a non-zero area of order $\mathcal{O}(\sqrt{\alpha}), \alpha \rightarrow 0$.

The reason of the non-uniformity can be found out by a scale transformation. Under the change $\rho=\sqrt{\alpha} \hat{\rho}, t=\tilde{t} / \sqrt{\alpha}$ we obtain the equation with the coefficients $\alpha=1, \lambda=(\mu / \alpha) \tilde{t}$. By this we see that as a small parameter, one should use the quotient $\mu / \alpha$, while as $\mu \approx \alpha$, there are no small parameters in the problem and it can not be simplified.

In what follows we consider system (1) and we focus the attention on the role of the additional parameter $\alpha$, which can not be removed by such simple transform in more complicated equations.

2.2. Adiabatic approximation. If the parameter $\mu$ is small, the coefficient $\lambda=\mu t$ varies slowly. The idea of the adiabatic approximation is to use a solution to the frozen system, in which $\lambda$ is supposed to be constant. Frozen system (1) possesses the first integral

$$
H(\rho, \varphi ; \lambda) \equiv \frac{(\rho-\lambda)^{2}}{2}-\alpha \cos \varphi=E=\mathrm{const}
$$

and is reduced to the pendulum equation by a shift in $\rho$. The solution can be written out:

$$
\rho=\lambda+\hat{\rho}(t, E), \quad \varphi=\hat{\varphi}(t, E) .
$$

Here $\hat{\rho}, \hat{\varphi}$ is the solution to the pendulum equations with the first integral being equal to

$$
\frac{\hat{\rho}^{2}}{2}-\alpha \cos \hat{\varphi}=E \text {. }
$$

In what follows we employ the family of periodic solutions (for the energies $-\alpha<E<\alpha$ ). Their frequency $\omega=\omega(E)$ depends on $E$. In terms of these functions we write out an approximate solution for the "unfrozen" problem with $\lambda=\mu t$.

In the formal constructions, it is convenient to employ functions with a fixed period:

$$
R(S, E)=\hat{\rho}\left(\frac{S}{\omega}, E\right), \quad \Phi(S, E)=\hat{\varphi}\left(\frac{S}{\omega}, E\right) .
$$

By the pendulum equation, these functions satisfy the following identities in the variables $S$, $E$ :

$\omega \partial_{s} R=\alpha \sin \Phi, \quad \omega \partial_{s} \Phi=-R, \quad \frac{R^{2}}{2}-\alpha \cos \Phi=E, \quad R \partial_{E} R+\alpha \sin \Phi \partial_{E} \Phi=1, \quad$ for all $S, E$.

In the original problem with the varying coefficient $\lambda=\lambda(t)$ we make the change of variables by the formulae

$$
\rho(t)=\lambda(t)+R(S(t), E(t)), \quad \varphi(t)=\Phi(S(t), E(t)) .
$$

For the new unknown functions $S(t), E(t)$ we obtain the equations

$$
\begin{aligned}
& \frac{d S}{d t}=\omega(E)-\lambda^{\prime}(t) \omega(E) \partial_{E} \Phi(S, E), \\
& \frac{d E}{d t}=\lambda^{\prime}(t) \omega(E) \partial_{S} \Phi(S, E) .
\end{aligned}
$$

If the coefficient $\lambda(t)$ varies slowly so that $\lambda^{\prime}(t)=\mathcal{O}(\mu), \mu \rightarrow 0$, then the leading terms of the asymptotics depend on the slow time:

$$
E=E_{0}(\tau)+\mathcal{O}(\mu), \quad S=\mu^{-1} S_{0}(\tau)+\mathcal{O}(1), \quad \mu \rightarrow 0, \quad \tau=\mu t .
$$


They are determined by the averaged equations

$$
\frac{d E_{0}}{d \tau}=0, \quad \frac{d S_{0}}{d \tau}=\omega\left(E_{0}\right)
$$

Thus, in the leading term of the asymptotics, the function $E(t)=$ const $+\mathcal{O}(\mu)$ remains constant (is an adiabatic invariant) for the times $0<\tau<\mathcal{O}(1)$ or in the fast scale $0<t<\mathcal{O}\left(\mu^{-1}\right)$.

In the adiabatic approximation, the solution is described in terms of the solution to the pendulum by the formulae:

$$
\rho(t)=\lambda(t)+\hat{\rho}\left(t, E_{0}\right)+\mathcal{O}(\mu), \quad \varphi=\hat{\varphi}\left(t+\mathcal{O}(1), E_{0}\right)+\mathcal{O}(\mu), \quad \mu \rightarrow 0, \quad 0<t<\mathcal{O}\left(\mu^{-1}\right) .
$$

As $\lambda=\mu t$, the approximate solutions have the ampltiude $\rho=\mu t+\hat{\rho}(t, E)+\mathcal{O}(\mu)$ slowly growing in time $\rho=\mu t+\mathcal{O}(1), t \rightarrow \infty$. The initial data, under which the functions $\hat{\rho}, \hat{\varphi}$ are bounded, correspond to the separatrix cell of the pendulum (7).

These formulae can make an impression that cell (7) is the domain of capture into resonance. However, while comparing it with the exact capture domain, we see that from the points near its boundary, non-resonance solutions with the slipping phase start. The numerical solutions to equations (1) is given in Figure 1. In the left, the solution with the initial point $(-2.2,0)$ in the approximate capture domain is given, it turns out to be non-resonance. In the right, we provide the resonance solution with the initial point $(-2,0)$ in the exact capture domain.

The differences between the domains are presented in Figures 4 and 5 . The difference between exact (6) and approximate (7) domains is of order $\mathcal{O}(\sqrt{\mu})$ as $\mu \rightarrow 0$ provided we fix $\alpha \neq 0$. If both $\mu \rightarrow 0$ and $\alpha \rightarrow 0$, then the width of the exact and approximate capture domains shrink and the width is of order $\mathcal{O}(\sqrt{\alpha})$. At the same time, the exact capture domain shifts and the error in the phase turns out to be of order one if $\alpha=\mathcal{O}(\mu)$.

As it was mentioned above, the proper small parameter is the quotient $\mu / \alpha$ and the difference between the capture domains is of order $\mathcal{O}(\sqrt{\mu / \alpha})$. Let us provide more precise results on this.

2.3. Error of capture domain in the adiabatic approximation. The stationary saddle point $\varphi_{s}$ of the original exact equation (5) is determined by the equation

$$
\sin \varphi=\frac{\mu}{\alpha} .
$$

The main term of its asymptotics is equal to $\pi$ and its determined the distance to the saddle point of the pendulum:

$$
\pi-\varphi_{s}=\frac{\mu}{\alpha}+\mathcal{O}\left(\left(\frac{\mu}{a}\right)^{2}\right), \quad \frac{\mu}{\alpha} \rightarrow 0 .
$$

The separatrix trajectory for equation (5) (as $\dot{\varphi}=\rho$ ) satisfies the relation

$$
\frac{1}{2} \rho^{2}-\alpha \cos \varphi-\mu \varphi=-\alpha \cos \varphi_{s}-\mu \varphi_{s}=\alpha-\mu \pi+\mu \mathcal{O}\left(\frac{\mu}{a}\right), \quad \frac{\mu}{\alpha} \rightarrow 0,
$$

which determines the exact boundary of the capture domain $\rho=\rho_{e x}(\varphi)$. The asymptotic description of these two branches of the boundary is given by the formula

$$
\rho_{\text {ex }}= \pm \sqrt{\alpha}\left[\sqrt{2(\cos \varphi+1)}+\frac{\mu}{\alpha} \frac{\varphi-\pi}{2 \sqrt{2(\cos \varphi+1)}}+\mathcal{O}\left(\left(\frac{\mu}{\alpha}\right)^{2}\right)\right], \quad \frac{\mu}{\alpha} \rightarrow 0 .
$$

The equation of the boundary of the approximate capture domain $\rho=\rho_{a s}(\varphi)$ corresponds to the separatrices of the pendulum

$$
\rho_{a s}= \pm \sqrt{\alpha} \sqrt{2(\cos \varphi+1)}
$$


The difference of these functions describes the "vertical gap" between the boundaries. For this gap we write the asymptotics:

$$
\left|\rho_{a s}(\varphi)-\rho_{e x}(\varphi)\right|=\sqrt{\alpha}\left[\frac{\mu}{\alpha} \frac{\pi-\varphi}{2 \sqrt{2(\cos \varphi+1)}}+\mathcal{O}\left(\left(\frac{\mu}{\alpha}\right)^{2}\right)\right], \quad \frac{\mu}{\alpha} \rightarrow 0,
$$

on the segment $\varphi_{0}<\varphi<\varphi_{s}$, where the separatrix loop of the original system is defined.

The left end-point $\varphi=\varphi_{0}$ of this segment is the intersection point of the separatrix loop and the axis $\rho=0$. It is defined by the equation

$$
-\alpha \cos \varphi-\mu \varphi=-\alpha \cos \varphi_{s}-\mu \varphi_{s}=\alpha-\mu \pi+\mu \mathcal{O}\left(\frac{\mu}{\alpha}\right), \quad \frac{\mu}{\alpha} \rightarrow 0,
$$

and its asymptotics determines the distance to $-\pi$ (the left saddle point of the pendulum)

$$
\pi+\varphi_{0}=\sqrt{\frac{\mu}{\alpha}} 2 \sqrt{\pi}+\mathcal{O}\left(\frac{\mu}{\alpha}\right), \quad \frac{\mu}{\alpha} \rightarrow 0 .
$$

Thus, on the most part of the segment $\varphi_{0}<\varphi<\varphi_{s}$ the distance between the boundaries is estimates by $\mathcal{O}(\mu / \alpha), \mu / \alpha \rightarrow 0$. However, in the vicinity of the turning point $\left(\rho=0, \varphi=\varphi_{0}\right)$ of the separatrix, the distance increases to $\mathcal{O}\left(\sqrt{\frac{\mu}{\alpha}}\right)$, Figures 4,5 . This result corresponds to the known estimate for the applicability domain of the averaging method [18].
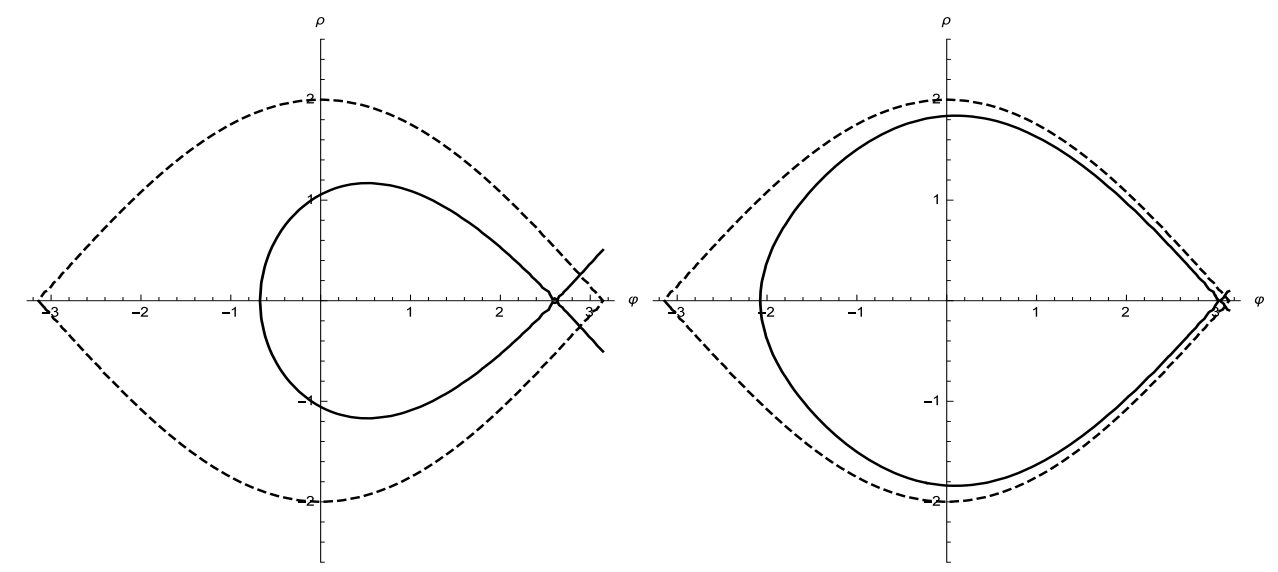

Figure 4. Separatrices of the pendulum (dashed line) border the domain of the capture into resonance in the adiabatic approximation. The separatrix loop (solid line) borders the exact domain of the capture into resonance. For the fixed $\alpha=1$ the difference depends on the parameter $\mu$. The left figure corresponds to $\mu=0.5$; the right one does to $\mu=0.1$.

The discussed asymptotic formulae make sense as $\mu / \alpha \rightarrow 0$. If both parameters are of the same order, $\mu / \alpha \approx 1$, then the discussion on closeness of the capture domains become wrong. Although both domains become narrow, $\rho^{2} \leqslant \alpha$, but there is a large change in the phase $\psi$ because of the shift of the point

$$
\varphi_{0}=-\pi+\mathcal{O}\left(\sqrt{\frac{\mu}{\alpha}}\right)
$$

The exact capture domain disappears at all as $\alpha<\mu$.

Conclusion. The adiabatic approximation is not applicable for describing the capture domain for small amplitude $\alpha \approx \mu$. 


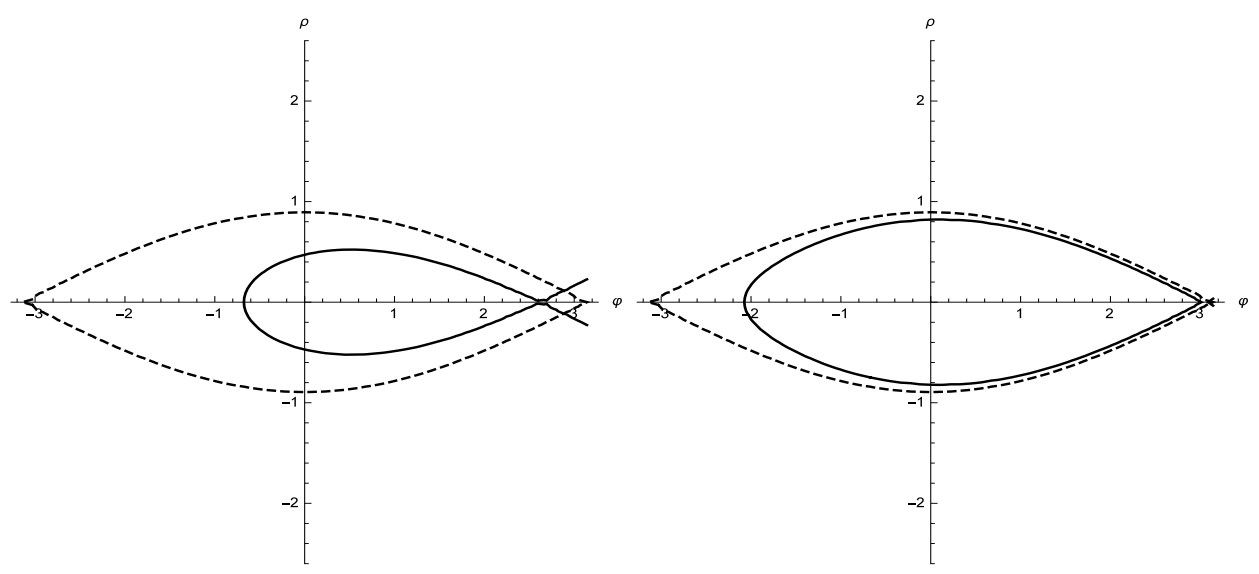

Figure 5. Separatrices of the pendulum (dashed line) border the domain of the capture into resonance in the adiabatic approximation. The separatrix loop (solid line) borders the exact domain of the capture into resonance. For small $a, \mu$ the difference depends on the quotient $\mu / \alpha$. The left figure corresponds to $\mu=0.1, \alpha=0.2$; the right one does to $\mu=0.02, \alpha=0.2$. This figure is in the same scale shrinking of as Figure 4.

In the considered example the non-integrability of original non-autonomous system (1) plays the crucial role for an exhausting analysis of the problem. This property allows us to describe the exact capture domain and compare it with the result of the asymptotic approximation.

\section{MAIN RESONANCE SYSTEM}

One more rather simple example is given by the known main resonance system (2). The coefficient $\lambda=\lambda_{0}+\mu t$ is supposed to depend on the slow time, that is, $0<\mu \ll 1$ is regarded as a small parameter. Moreover, the positive coefficient $\alpha>0$ can be also small. The studied issue is on resonance solutions with an unboundedly growing amplitude $r(t)$. The main aim is as follows: to find out the ineligibility of the adiabatic approximation for small $\alpha$ in the problem on determining the capture domain.

The constant $\lambda_{0}=$ const $>0$ is taken fixed and positive. Without loss of generality we can assume that $\lambda_{0}=1$ since the equations are reduced to this case by the renormalization of the variables $r=\tilde{r} \sqrt{\lambda_{0}}, t=\tilde{t} / \lambda_{0}$ and other coefficients $\alpha=\tilde{\alpha} \lambda_{0}^{3 / 2}, \mu=\tilde{\mu} / \lambda_{0}^{2}$. In what follows we omit the tilde.

The problem with $\lambda=1+\mu t$ allows us to reduce a little the bulkiness of the formulae and clarify better the role of the remaining pair of the parameters $\mu, \alpha$. For equation (2), the existence of the two-parametric family of resonance solutions with an unboundedly growing amplitude $r(t)$ is known [8]. However, the set of the initial points for such solutions, the domain of the capture into resonance, remains unknown.

3.1. Frozen system. The autonomous (frozen) system with $\lambda=$ const has the first integral

$$
J(r, \psi) \equiv \frac{1}{4} r^{4}-\frac{1}{2} \lambda r^{2}+\alpha r \sin \psi=\text { const. }
$$

The structure of the phase portrait (the level lines of the first integral) depends on the parameters $\alpha, \lambda$, see Figure 6. No resonance solutions exist in the autonomous system.

3.2. Adiabatic approximation. If the parameter $\mu$ is small, the adiabatic approximation is possible. It is based on the solution of the frozen system. The asymptotic construction is made by the averaging method similar to the previous examples; the details are given in work 
[19]. Under this approximation, a part of the capture domain can be identified. It is bordered by the separatrices loops taken in the phase portrait for the initial value of $\lambda$; in the considered case this is $\lambda=1$. The capture domain determined in this way (more precisely, its determined part) depends on the parameter $\alpha$. In Figure 7 we provide examples for various $\alpha$. Here we do not discuss the capture intro resonance from the set of the points located outside the separatrix loop, which is described in probability terms [18].
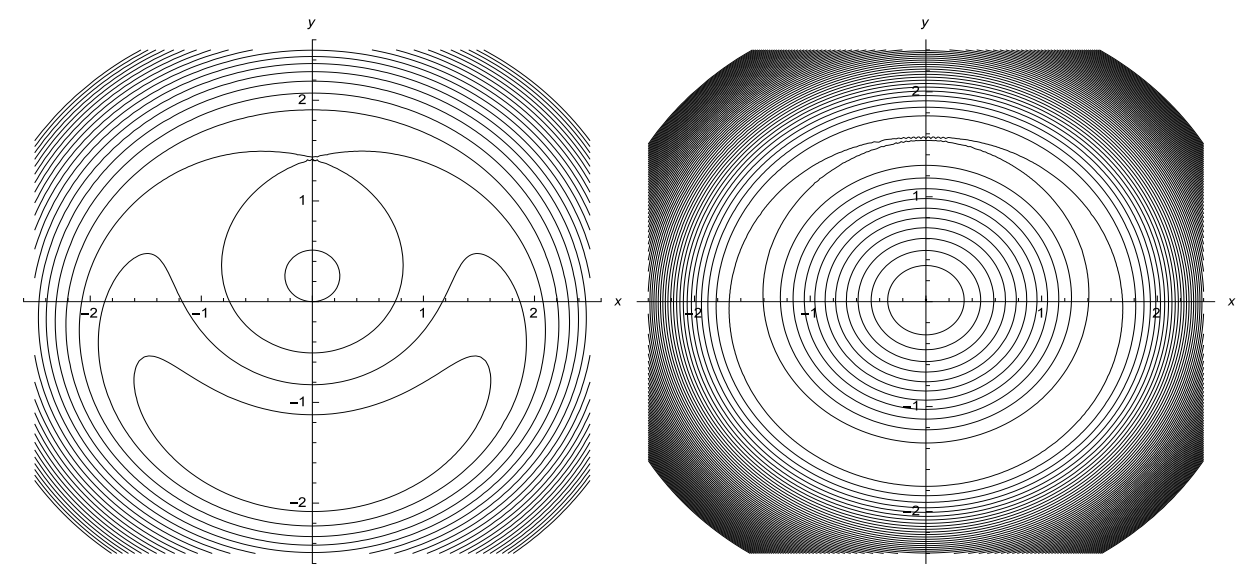

Figure 6. Phase portrait of the frozen main resonance system depends on the parameters $\alpha$ and $\lambda$.

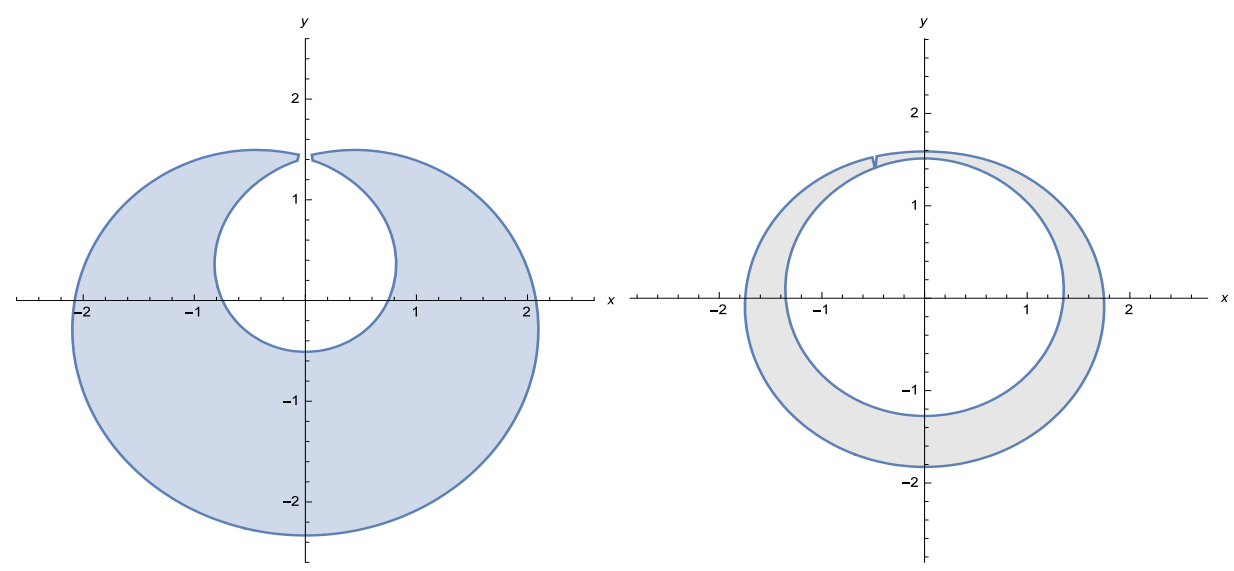

FiguRE 7 . The domain of the capture into resonance for the main resonance system in the adiabatic approximation is found by the phase portrait for the initial value of $\lambda$. This domain shrinks as the parameter $\alpha$ becomes smaller.

Under the variation of the coefficient $\lambda$, the phase portrait is deformed. In particular, the separatrices loops goes to infinity as $\lambda$ grows. At the same time, the closed trajectories corresponding to the periodic solutions of the frozen sytstem are shifted. The exact trajectories for the unfrozen system look like spirals. They are approximately described by means of the averaged system. The area enveloped by such approximated trajectory is constant in the leading term of the asymptotics in the small parameter $\mu \rightarrow 0$. In this case the area is an adiabatic invariant and the corresponding approximation is called adiabatic. Since the area between the separatrices grows as $\lambda$ does, the trajectories starting from the capture domain slowly goes to the infinity. This phenomenon is identified as the capture into the resonance.

However, one should keep in mind that such approximation does not always corresponds to an exact solution. The solutions starting near the boundary of the mentioned approximated 
capture domain can turn out to be non-resonance. The width of such boundary strip is estimated as $\mathcal{O}(\sqrt{\mu}|\ln \mu|)$ as $\mu \rightarrow 0$, [18]. This means that the capture into resonance is ensured by a narrower domain in comparison with that between the separatrices. Moreover, the description of the capture domain in the adiabatic approximation can turn out to be wrong at all if the distance between the separatrices is too small. Exactly such phenomenon is observed for small $\alpha$. Here the matter is not in the estimates for the error, which can be rough. Numerical experiments show that the trajectories starting from a narrow capture domain are mostly non-resonance. The estimates of the width of the boundary strip, for which the capture into resonance is not ensured, are known in terms of the adiabatic parameter $\mu$ [18]. In that follows we provide the estimates of the width of the capture domain in terms of the parameter $\alpha$. All this allows us to formulate restrictions for the pair of the parameters $\alpha, \mu$ ensuring that the boundary strip covers the entire domain and the capture into resonance between the separatrices is not guaranteed.

Theorem 1. As $\lambda=1$, the distance between the separatrices has the asymptotics

$$
2 \sqrt{\alpha}[1+\mathcal{O}(\alpha)] \quad \text { as } \quad \alpha \rightarrow 0 .
$$

Proof. The stationary points of the system are located on the rays of the angle $\psi= \pm \frac{\pi}{2}$. Their radius-coordinates are determined by the equations $r^{3}-r \pm \alpha=0$. This implies easily the asymptotics for the radius-coordinates of the saddle $r_{+}(\alpha)$ in the upper half-plane and the center $r_{-}(\alpha)$ in the lower half-plane

$$
r_{ \pm}(\alpha)=1 \mp \frac{\alpha}{2}+\mathcal{O}\left(\alpha^{2}\right), \quad \alpha \rightarrow 0
$$

For the values of the first integral $J_{ \pm}$at these points we obtain the relation

$$
J_{ \pm}=J\left(r_{ \pm}, \pm \pi / 2\right)=\frac{1}{4} r_{ \pm}\left[r_{ \pm}^{3}-2 r_{ \pm} \mp 4 \alpha\right]=\frac{1}{4} r_{ \pm}\left[ \pm 3 \alpha-r_{ \pm}\right]
$$

and for the difference we get the asymptotics

$$
J_{+}-J_{-}=-\frac{1}{4}\left[\left(r_{+}+r_{-}\right) 3 \alpha+r_{+}^{2}-r_{-}^{2}\right]=\alpha+\mathcal{O}\left(\alpha^{2}\right) .
$$

We consider the equation for the separatrices $J(r, \psi)=J_{+}$on the ray $\psi=-\frac{\pi}{2}$ and employ the Taylor expansion at the stationary point $r_{-}$:

$$
J_{-}+\frac{1}{2} J_{r r}\left(r_{-},-\frac{\pi}{2}\right)\left(r-r_{-}\right)^{2}+\mathcal{O}\left(r-r_{-}\right)^{3}=J_{+}
$$

Since

$$
J_{r r}\left(r_{-},-\frac{\pi}{2}\right)=3 r_{-}^{2}-1=2+\mathcal{O}(\alpha)
$$

we obtain

$$
\left(r-r_{-}\right)^{2}[1+\mathcal{O}(\alpha)]+\mathcal{O}\left(r-r_{-}\right)^{3}=J_{+}-J_{-}=\alpha+\mathcal{O}\left(\alpha^{2}\right) .
$$

Therefore, the distance from the center to the separatrix is $\left|r-r_{-}\right|=\sqrt{\alpha}[1+\mathcal{O}(\alpha)]$. This implies the asymptotic estimate for the distance between the separatrices.

In the same way we estimate the distance over other rays. At the same time, the monotonicity of the distance between the separatrices in the angle is seen in the phase portrait.

Corollary 1. If the parameter $\alpha$ is not too small, $\alpha \gg \mathcal{O}(\mu|\ln \mu|), \mu \rightarrow 0$, then the method of adiabatic approximation in the small parameter $\mu \rightarrow 0$ is applicable for system (2). The boundaries of the capture domain are approximately described by the separatrices of the frozen system as $t=0$ [18]. 
Corollary 2. If the parameter $\alpha$ is too small, $\alpha \leqslant \mathcal{O}(\mu), \mu \rightarrow 0$, then the method of the adiabatic approximation in the small parameter $\mu \rightarrow 0$ is not applicable and the capture domain is not determined by the separatrices of the frozen system.

These corollaries are implied by the estimate of the width of the boundary strip by $\mathcal{O}(\mu|\ln \mu|)$ and by estimate (8).

3.3. Approximation by the shifted pendulum. It follows from estimate (8) that the capture domain shrinks as $\alpha$ becomes smaller. This effect exactly corresponds to what was demonstrated in the previous example. In that simpler problem we knew the location of the capture domain. The error of the adiabatic approximation was found by the comparison. This estimate turned out to be large if the parameter $\alpha$ is small, for instance, as $\alpha=\mathcal{O}(\mu)$. Moreover, we found out that as $\alpha=\mathcal{O}(\mu)$, by a scale transform the original system is reduced to equations (1) with $\alpha=1, \mu=\mathcal{O}(1)$ (or, the same, to shifted pendulum (5)) with no small parameters. The exact capture domain was bounded by the separatrix loop of such system.

System (2) considered here is more complicated and we can not exclude the parameter by a scale transform. However, such procedure can be done in the asymptotic approximation. In this way, in the leading term of the asymptotics the same shifted pendulum (5) rises, which in the case $\alpha=\mathcal{O}(\mu)$ involves not small parameters. The separatrix loop for such system determines the location of the capture domain in the asymptotic approximation as $\alpha \approx \mu \rightarrow 0$.

Theorem 2. If the small parameters $\alpha$, $\mu$ obey the relation $\mathcal{O}(\mu) \leqslant \alpha \ll 1$, then the capture domain is narrow of order $\mathcal{O}(\sqrt{\alpha})$. Its boundary is approximately described by the separatrix loop of the shifted pendulum corresponding to the system

$$
\frac{d \rho}{d \tau}=\sin \psi, \quad \frac{d \psi}{d \tau}=\nu \tau-\rho, \quad \tau>0
$$

with the coefficient $\nu=\mu / 2 \alpha$ and $\tau=\sqrt{2 \alpha} t$.

Proof. If the parameter $\alpha \ll 1$ is small, at the initial moment, the separatrices of frozen system (2) are located near the circumference $r=1$. This is an attempt to specify the location of narrow (for small $\alpha$ ) capture domain near this line looks natural. In order to do it, we make a shift and a scale transform of the amplitude

$$
r(t)=1+\sqrt{\frac{\alpha}{2}} \rho(\tau), \quad \psi=\varphi-\frac{\pi}{2}, \quad \tau=\sqrt{2 \alpha} t .
$$

After such change we obtain system

$$
\frac{d \rho}{d \tau}=\sin \psi, \quad \frac{d \varphi}{d \tau}+\rho-\nu \tau=\sqrt{\alpha}\left[\frac{1}{\sqrt{2}+\sqrt{\alpha} \rho} \cos \psi-\frac{1}{2 \sqrt{2}} \rho^{2}\right], \quad \tau>0,
$$

with the coefficient $\nu=\frac{\mu}{2} \alpha$. After that we pose the problem on finding a solution with an unboundedly growing function $\rho(\tau)$. The problem is solved by the asymptotic approximation in the parameter $\alpha \rightarrow 0$ under the condition that the second parameter $\nu \leqslant$ const $<\infty$ is not large. For the leading terms of the asymptotics

$$
\rho(\tau)=\rho_{0}(\tau)+\mathcal{O}(\sqrt{a}), \quad \varphi(\tau)=\varphi_{0}(\tau)+\mathcal{O}(\sqrt{a})
$$

we obtain the system (9), which is reduced to shifted pendulum (5). The separatrix loop in the phase portrait of this equation exists as $|\nu|<1$ and describes the capture domain in this approximation. It is obvious that as $|\alpha|<\frac{\mu}{2}$, such domain does not exist.

If the parameter $\alpha$ is not too small, $|\alpha| \gg \mu$, then in the equation of the shifted pendulum the parameter $\nu$ turns out to be small. Here again the adiabatic approximation as $\nu \rightarrow 0$ is possible. The obtained in this way capture domain coincides with the cell bounded by the separatrices 


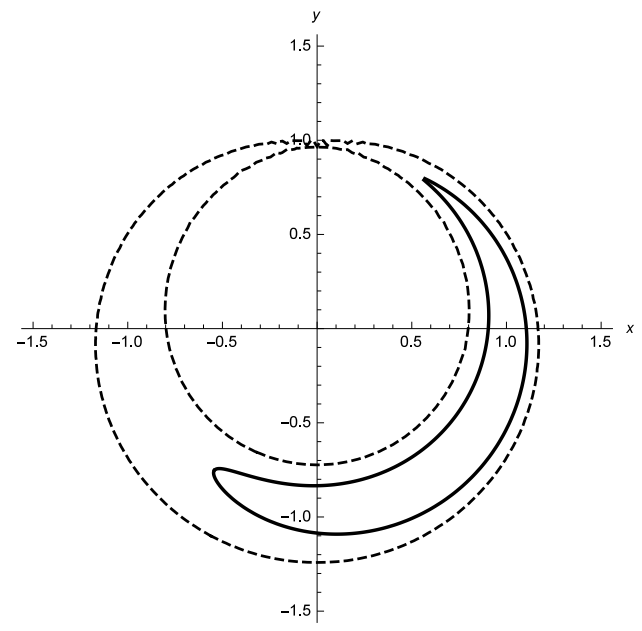

FIGURE 8. Differences in determining the capture domain between the adiabatic approximation (dashed line) and the approximation by the shifted pendulum (solid line) for small $\alpha \approx \mu \ll 1$. The result is similar to Figure 5 .

of the pendulum. Taking into consideration the conditions for passing to the shifted pendulum, we obtain the restrictions $\mu \ll|\alpha| \ll 1$.

Thus, the plane of the parameters $\alpha, \mu$ is split into several sectors in the vicinity of the zero. In each of these sectors, the capture domain is described by different formulae. These formulae coincide asymptotically in the intersections of the sectors. Moreover, in the intersection we can write out a separate formula obtained in the adiabatic approximation for system (9). These results correspond to the ideas in matching of asymptotic expansions [3, which are usually applied in different domains of independent variables.

3.4. Approximation of main resonance. original system (2) with $\lambda=\lambda_{0}+\mu t$ involves one more parameter $\lambda_{0}$. Up to now, this parameter has been assumed to be a fixed positive constant. However, if this parameter is small or is zero, the above given analysis is not applicable. In particular, the adiabatic approximation does not determine the capture domain at least since as $\lambda=0$, the phase portrait contains no separatrices. In this case it is convenient to renormalize the variables excluding one of the parameters: $t=\alpha^{-\frac{2}{3}} \tau, r=\alpha^{\frac{1}{3}} R(\tau)$. As a result, for new functions $R, \psi(\tau)$ we obtain the system

$$
\frac{d R}{d t}=\cos \psi, \quad R\left[\frac{d \psi}{d t}+R^{2}-\Lambda\right]=-\sin \psi, \quad t>0
$$

with the coefficient $\Lambda=\lambda_{0} \alpha^{-\frac{2}{3}}+\mu \alpha^{-\frac{4}{3}} \tau$.

The parameter $\mu \alpha^{-\frac{4}{3}} \ll 1$ in this coefficient is small and $\lambda_{0} \alpha^{-\frac{2}{3}} \leqslant \mathcal{O}(1)$ is not large, the adiabatic approximation is possible. The capture domain is approximately determined by $\lambda_{0} \alpha^{-\frac{2}{3}}$. This approximation can be ineligible if $\lambda_{0}$ is too small.

If the parameter $\mu \alpha^{-\frac{4}{3}}=\mathcal{O}(1)$ is not small and $\lambda_{0} \alpha^{-\frac{2}{3}} \leqslant \mathcal{O}(1)$ is not large, then the adiabatic approximation is impossible. In this case complete main resonance system 10 is a model problem, which can not be further simplified. The capture domain is determined in numerical experiments.

\section{Asymptotic APPRoximation FOR GENERAL SYSTEM}

A similar situation holds for more general equations (3), (4). Here non-autonomous system is not integrable, too, and there is no information on exact capture domain. 


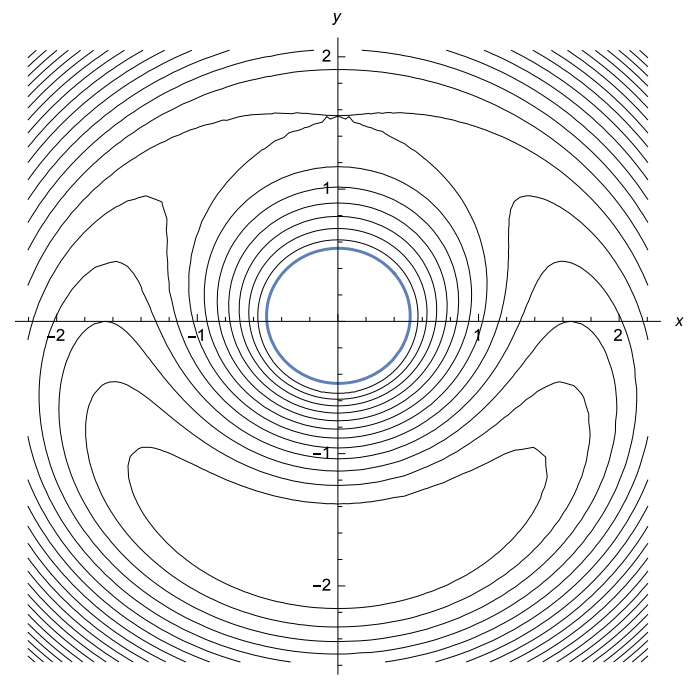

Figure 9. Phase portrait of frozen system (3), (4)

The analysis of the problem in the adiabatic approximation is based on the first integral for the frozen system

$$
Z(\gamma, \psi) \equiv \frac{1}{2}(\gamma-\lambda)^{2}+\alpha \sqrt{\gamma^{2}+2 b \gamma-d} \sin \psi=\text { const. }
$$

The phase portrait is similar to main resonance system. The phase trajectories except a pair of separatrices are closed curs in the plane with polar coordinates $\psi, \gamma$. The domain of the capture into resonance is located between the separatrices loops. This domain shrinks as $\alpha \rightarrow 0$. The detailed study of a narrow capture domain was provided in [22].

We note that the specifying the location of a narrow capture domain leads one either to the problem for the shifted pendulum or to the main resonance system [22]. Such results correspond to the known works by physicists in [13], [14]. They explain the reason why the adiabatic approximation was ignored in these and other works, for instance, in [20], [21].

\section{Conclusion}

The problems on capture into resonance can be approximately studied by the averaging method if the original differential equations involve slowly varying coefficients. In the general situation this approximation is not uniform w.r.t. other parameters involved in the equation. The condition of applicability of the used approximation are found out while analyzing the role of additional parameters. The critical values of the parameters turn out to be those, for which the approximate capture domain collapses into the line. In the vicinity of such values, another form of averaging method is employed, which in the leading term of the asymptotics leads either to the shifted pendulum equation or to the main resonance system. The issue on how exact is the description of the capture domain in such approach remains open.

\section{BIBLIOGRAPHY}

1. V.M. Babich, V.S. Buldyrev. The art of asymptotics // Vestn. Leningr. Univ. Mat. Mekh. Astron. 13:3, 5-12 (1977). (in Russian).

2. I.V. Andrianov, R.G. Barantsev, L.I. Manevich. Asymptotic mathematics and synergetics. URSS, Moscow (2004). (in Russian).

3. A.M. Il'in. Matching of asymptotic expansions of solutions of boundary value problems. Nauka, Moscow (1989). [Trans. Math. Monog. 102. Amer. Math. Soc., Providence (1992).] 
4. N.N. Bogolyubov, Yu.A. Mitropol'sky. Asymptotic methods in the theory of non-linear oscillations. Nauka, Moscow (1974). [Gordon and Breach, New York (1961)].

5. V.I. Arnold, V.V. Kozlov, A.I. Neishtadt. Mathematical aspects of classical and celestial mechanics. VINITI, Moscow (1985). [Springer, Berlin (1997).]

6. J. Brüning, S.Yu. Dobrokhotov, M.A. Poteryakhin. Averaging for Hamiltonian systems with one fast phase and small amplitudes // Matem. Zamet. 70:5, 660-669 (2001). [Math.l Notes. 70:5, 599-607 (2001).]

7. B.V. Chirikov. The passage of a nonlinear oscillating system through resonance // Dokl. Akad. Nauk SSSR. 125:5, 1015-1018 (1959). [Sov. Phys. Dokl. 4, 390-394 (1959).]

8. L.A. Kalyakin. Asymptotic behavior of solutions of equations of main resonance // Teor. Matem. Fiz. 137:1, 142-152 (2003). [Theor. Math. Phys. 137:1, 1476-1484 (2003).]

9. L.A. Kalyakin. Asymptotic analysis of autoresonance models // Uspekhi Matem. Nauk. 63:5, 3-72. [Russ. Math. Surv. 63:5, 791-857 (2008).]

10. L.D. Landau, E.M. Lifshitz. Course of theoretical physics. Vol. 2. The classical theory of fields. Nauka, Moscow (1973). [Butterworth-Heinemann, Burlington (1975).]

11. V P Milant'ev. Cyclotron autoresonance - 50 years since its discovery // Uspekhi Fiz. Nauk. 180:8, 875-884 (2013). [Physics-Uspekhi. 56:8, 823-832 (2013).]

12. L.A. Kalyakin. Asymptotic analysis of the model of cyclotron gyromagnetic autoresonance // Vestnik ChelGU. Fiz. 21, 68-74 (2015). (in Russian).

13. K.S. Golovanivsky. Autoresonant acceleration of electrons at nonlinear ECR in a magnetic field which is smoothly growing in time // Physica Scripta. 22:2, 126-133 (1980).

14. K.S. Golovanivsky. The gyromagnetic autoresonance // IEEE Trans. Plasma Sci. 11:1, 28-35 (1983).

15. A.P. Itin, A.I. Neishtadt, A.A. Vasiliev. Capture into resonance in dynamics of a charged partice in magnetic field and electrostatic wave // Physica D. 141:4, 281-296 (2000).

16. R. Haberman, E.K. Ho. Boundary of the basin of attraction for weakly damped primery resonance // J.Appl. Mech. 62:3, 941-946 (1990).

17. O.M. Kiselev and S.G. Glebov. An asymptotic solution slowly crossing the separatrix near a saddle-centre bifurcation point // Nonlinearity. 16:1, 327-362 (2003).

18. A.I. Neishtadt. Passage through a separatrix in a resonance problem with a slowly-varying parameter // Prikl. Matem. Mekh. 39:4, 621-632 (1975). [J. Appl. Math. Mech. 39:4, 594-605 (1975).]

19. L.A. Kalyakin. Asymptotic analysis of an autoresonance model // Russ. J. Math. Phys. 9:1, 84-95 (2002).

20. T. Armon and L. Friedland. Chirped resonance dynamics in phase space // J. Plasma Phys. 82:5, 705820501 (2016).

21. T. Armon and L. Friedland. Capture into resonance and phase-space dynamics in an optical centrifuge // Phys. Rev. A. 93:4, 043406 (2016).

22. L.A. Kalyakin. Asymptotic analysis of the model of gyromagnetic autoresonance // Zhurn. Vychisl. Matem. Matem. Fiz. 57:2, 285-301. [Comput. Math. Math. Phys. 57:2, 281-296 (2017).]

Leonind Anatol'evich Kalyakin

Institute of Mathematics, Ufa Scientific Center, RAS,

Chernyshevsky str. 112,

450008, Ufa, Russia

E-mail: klenru@mail.ru 